\title{
Complicações pós-operatórias precoces de trabeculectomia com mitomicina, em pacientes portadores de glaucoma primário de ângulo aberto
}

\author{
Precocious postoperative complications \\ of mitomicina trabeculectomy, in carrying patients \\ with primary open angle glaucoma
}

Gustavvo Carneiro Vilasboas Gutemberg ${ }^{1}$, Fernando José da Silva Filho², José Ricardo Carvalho Lima Rehder

\section{ResUmo}

Objetivo: Avaliar as complicações precoces encontradas em cirurgias de trabeculectomia com uso de mitomicina, realizadas em serviço-escola de residência médica, visando melhor aperfeiçoamento na técnica e acompanhamento pós-operatório dos pacientes. Métodos: Estudo retrospectivo por meio de análise de prontuários. Os pacientes foram avaliados quanto ao sexo, idade e raça. Foram incluídos todos os pacientes portadores de glaucoma primário de ângulo aberto e submetidos à trabeculectomia com mitomicina. Um total de 43 pacientes foram analisados, 25 eram do sexo masculino, 18 do sexo feminino; a idade variou de 51 a 82 anos (média 68 anos) e 19 eram negros. Os pacientes eram avaliados no $1^{\circ}$ e $7^{\circ}$ dia de pós-operatórios, ou se necessário antes da $1^{\text {a }}$ semana. As complicações foram registradas e constituíam de: vazamento da bolha; câmara anterior rasa; atalamia; h; Descolamento de coróide. Resultados: A média de idade foi de 68 anos, $58,1 \%$ dos pacientes ( 25 no total) eram do sexo masculino, $44,1 \%$ eram negros. O vazamento da bolha foi a intercorrência mais frequente, representando $25,56 \%$ das cirurgias e $36,66 \%$ de todas as complicações (11 pacientes). Câmara anterior rasa foi observada em $17,5 \%$ das cirurgias e $22,5 \%$ das complicações (sete pacientes). Descolamento de coróide esteve presente em 14,6\% dos pacientes e 19,3\% das complicações na $1^{a}$ semana (sete pacientes). Atalamia foi encontrada em três pacientes, representou de $6,97 \%$ dos pacientes e $9,67 \%$ das complicações. Hifema também foi observado em três pacientes, sendo todos eles encontrados no pós-operatório imediato. Conclusão: A frequência de complicações encontrados neste trabalho está dentro dos limites observados na literatura. Quantificar o percentual de complicações pós-operatórias durante a primeira semana é de fundamental importância para melhorias na técnica intraoperatória e padronização das condutas frente a cada complicação, diminuindo assim a morbidade e a chance de falência cirúrgica a longo prazo.

Descritores: Glaucoma de ângulo aberto/cirurgia; Complicações pós-operatórias; Trabeculectomia/efeitos adversos; Mitomicina/uso terapêutico

\footnotetext{
'Estagiário da Faculdade de Medicina do ABC - FMABC - Santo André (SP), Brasil;

${ }^{2}$ Médico colaborador do Setor de Glaucoma da Faculdade de Medicina do ABC - FMABC - Santo André (SP), Brasil;

${ }^{3}$ Professor Titular da Disciplina de Oftalmologia da Faculdade de Medicina do ABC - FMABC - Santo André (SP), Brasil;

Disciplina de Oftalmologia da Faculdade de Medicina do ABC - FMABC - Santo André (SP), Brasil.

Recebido para publicação em: 22/09/2009 - Aceito para publicação em 26/01/2010
}

Rev Bras Oftalmol. 2010; 69 (2): 100-3 


\section{INTRODUÇÃO}

$\mathbf{0}$ glaucoma primário de ângulo aberto é uma neuropatia óptica crônica progressiva que tem como principal fator de risco o aumento da pressão intraocular. É caracterizado por alterações típicas do disco óptico e da camada de fibras nervosas da retina ${ }^{(1)}$. É a principal causa de cegueira irreversível no mundo. A trabeculectomia tem sido a cirurgia mais realizada para o tratamento do glaucoma não controlado clinicamente. Descritas pela primeira vez há mais de uma década, o uso de mitomicina tem se tornado o mais popular adjuvante na cirurgia filtrante ${ }^{(3)}$. A trabeculectomia, associada ao uso de antifibroblásticos, como a mitomicina, tem sido indicado não somente em casos onde há maior risco de falência cirúrgica, mas também em glaucoma primário de ângulo aberto simples, devido ao menor risco de fibrose da bolha a longo pra$\mathrm{zo}^{(2)}$. Em parte, isso pode ser atribuído à fácil aplicação quando comparado com o tratamento com Fluorouracil ${ }^{(3)}$. Pacientes jovens e/ou com ancestrais negros podem apresentar maior risco de falência. O objetivo da cirurgia filtrante é reduzir e manter a pressão intraocular em um nível que irá prevenir maior dano ao nervo óptico e no campo visual. O índice de sucesso da trabeculectomia nos casos de glaucoma primário de ângulo aberto varia de 80 a $90 \%{ }^{(2)}$, porém existe um índice significativo de complicações pós-operatórias que geram grande morbidade, necessitando de frequente acompanhamento pós-operatório.

\section{Métodos}

Este é um estudo retrospectivo por meio de análise de prontuários de pacientes registrados no setor de glaucoma do serviço de oftalmologia da Faculdade de Medicina do $\mathrm{ABC}$, submetidos à cirurgia no período de 21 de janeiro a 18 setembro de 2008 . Os pacientes avaliados quanto ao sexo, idade e raça. Foram incluídos todos os pacientes portadores de glaucoma primário de ângulo aberto em uso ou não de medicação hipotensora, submetidos à trabeculectomia com mitomicina. Foram excluídos pacientes com glaucomas secundários, submetidos à cirurgia filtrante prévia ou procedimentos que manipulavam conjuntiva superior. Dos 43 pacientes analisados, 25 eram do sexo masculino, 18 do sexo feminino, e 19 eram negros e a idade variou de 51 a 82 anos (média 68 anos). A indicação de trabeculectomia era basicamente devido à manutenção de pressão intraocular elevada apesar de uso de medicação máxima ou em alguns casos pela condição social que impedia o uso regular dos colírios. A cirurgia realizada se constituiu dos passos a seguir:

- Anestesia peribulbar ou subtenoniana com lilocaína $2 \%$ sem vasoconstrictor;

- Corneopexia superior com fio seda 6.0;

- Abertura conjuntival superior base fórnice;

- Confecção de flap escleral quadrangular de 3x3mm;

- Aplicação de esponjas embebidas com mitomicina $0,02 \%$ por 3 minutos;

- Lavagem com solução salina balanceada $10 \mathrm{ml}$.

Paracentese acessória

- Incisão principal na altura do limbo;

- Trabeculectomia com trabeculótomo;

- Iridectomia periférica com tesoura de Vannas;

- Fechamento do flap escleral com 2 pontos simples nylon

10.0 ;

- Fechamento conjuntival com 2 pontos contínuos nylon 10.0

- Observação da bolha e teste de Seidel;

- Atropina 1\% 01 gota 15-Curativo oclusivo.

Os pacientes eram avaliados no $1^{\circ}$ e $7^{\circ}$ dia pósoperatórios, ou se necessário antes da $1^{\mathrm{a}}$ semana. As complicações não foram registradas e constituíam de: Vazamento da bolha (Teste de Seidel positivo) com ou sem necessidade de ressutura, câmara anterior rasa, Atalamia (casos com toque iririano ou iridocristaliniano), Hifema, descolamento de coróide (avaliado somente na $1^{\mathrm{a}}$ semana)

\section{Resultados}

A média de idade foi de 68 anos (53-82), 58,1\% dos pacientes ( 25 no total) eram do sexo masculino, $41,9 \%$ eram do sexo feminino. Dentre elas, o vazamento da bolha foi a intercorrência mais frequente, dos 43 pacientes um total de 11 apresentavam Seidel, 10 no pós-operatório imediato e 04 na $1^{\mathrm{a}}$ semana (com apenas um caso novo), representando $25,56 \%$ das cirurgias e $36,66 \%$ de todas as complicações. A necessidade de ressutura somente em quatro casos sendo apenas um no POI. Câmara anterior rasa foi observado em sete pacientes, sendo todos já observados desde o $1^{\circ}$ pós-operatório representando um total de $17,5 \%$ das cirurgias e $22,5 \%$ das complicações. Descolamento de coróide esteve presente em seis pacientes na $1^{\text {a }}$ semana, correspondendo a $14,6 \%$ dos pacientes e $19,3 \%$ das complicações. Atalamia foi encontrada em três pacientes, um com toque iridocorneano periférico e dois 
com toque iriano central que se mantiveram atalâmicos até a primeira semana, representa um total de 6,97 e $9,67 \%$ quanto aos casos e complicações respectivamente, sendo necessária intervenção cirúrgica em 01 destes pacientes. Hifema também foi observado em três pacientes, sendo todos eles identificados no pós-operatório com melhora espontânea durante a primeira semana.

\section{DısCUSSÃO}

A trabeculectomia vem sendo empregada como cirurgia de eleição em pacientes com glaucoma. Chen ${ }^{(3,4)}$ introduziu o uso de mitomicina como quimioterapia adjuvante durante a trabeculectomia em 3 olhos com glaucoma refratário.

A mitomicina atua inibindo a proliferação fibroblástica e fibrose subconjuntival, diminuindo cicatrização da bolha. No momento atual existe uma tendência para o uso de antimetabólicos para a maioria dos procedimentos cirúrgicos fistiluzantes, pois comprovadamente o benefício, pelo aumento nas taxas de trabeculectomias funcionantes a longo prazo tem superado o risco do aumento de complicações.

Existem poucos trabalhos na literatura que avaliam detalhadamente as complicações operatórias durante a primeira semana de cirurgia, focando basicamente no acompanhamento a médio e longo prazo dos pacientes quanto ao sucesso da trabeculectomia. Quantificar essas complicações e discutir possíveis condutas é de suma importância para diminuir a morbidade e aumentar a chance de patência a longo prazo do procedimento fistulizante.

O vazamento da bolha se destacou entre todas as complicações, correspondendo a $25,56 \%$ das cirurgias e $36,66 \%$ de todas as complicações. Dados da literatura variam muito quanto ao aparecimento de seidel, variando de 5,6 a $40 \%$ dos procedimentos ${ }^{(5)}$. No seguimento destes casos a conduta varia conforme o tipo e quantidade de vazamento, incluindo desde aplicação de colírios hipotensores e diminuição do uso de corticóde, oclusão, LC terapêutica até de intervenção cirúrgica para oclusão do vazamento ${ }^{(6)}$.

Hipotonia foi observada em sete pacientes, sendo todos já observados desde o $1^{\circ} \mathrm{PO}$, representando um total de $17,5 \%$ das cirurgias e $22,5 \%$ das complicações. Achado muito freqüente no POI de trabeculectomias, sendo mais importante no $2^{\circ}$ e $3^{\circ}$ dias $^{(6)}$, pode ser considerada como evolução natural do procedimento fistulizante, contanto que, não se observe persistência

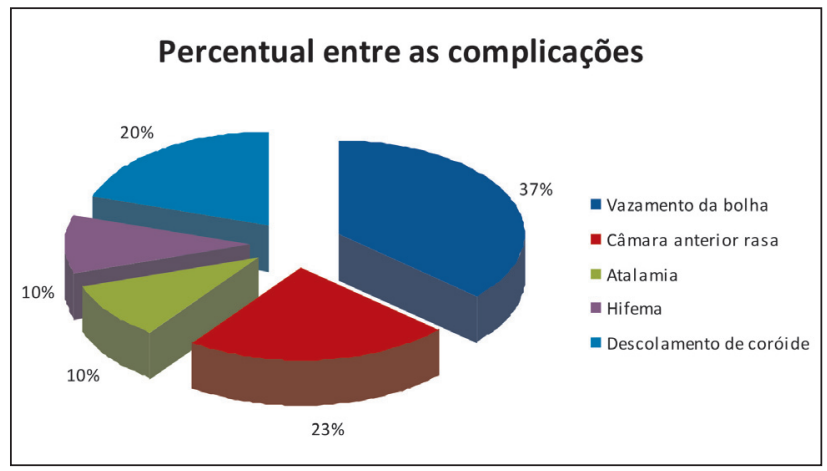

por mais de 02 semanas devido ao risco de maculopatia hipotônica $^{(7)}$.

A presença de decolamentos coroidianos aparece com frequência nos pós-operatórios de trabeculectomia, variando de 5 a $40 \%{ }^{(8)}$. Foi encontrado em seis pacientes na $1^{\mathrm{a}}$ semana, correspondendo a $14,6 \%$ dos pacientes e 19,3\% das complicações. A maioria dos casos dessa afecção é transitória e evolui com melhora espontânea entre o $10^{\circ} \mathrm{e} 14^{\circ}$ dia.

Atalamia representa um total de $6,97 \%$ e $9,67 \%$ quanto aos casos e complicações, respectivamente. Dos três pacientes referidos apenas um apresentou toque iridocristaliniano, observado no $7^{\circ} \mathrm{PO}$. Nestes casos, é mandatório intervenção imediata pelo risco de descompensação corneana, formação de catarata, falência da bolha e formação de sinéquias anteriores e posteriores importantes. Os dois pacientes restantes foram observados durante a $1^{\mathrm{a}}$ semana com melhora progressiva da profundidade da câmara anterior.

O hifema se apresentou com a mesma incidência de atalamia. A tendência nestes casos é de melhora espontânea durante as primeiras 24 a 48 horas, como foi observado nos pacientes do trabalho.

Percentual de complicações encontra-se no gráfico1.

\section{Conclusão}

A cirurgia de trabeculectomia com mitomicina é hoje o procedimento de escolha na maioria dos casos de glaucomas primários de ângulo aberto. O índice de complicações encontrados neste trabalho está entre os limites observados na literatura. Identificar e quantificar o percentual de complicações pós-operatórias durante a primeira semana é de fundamental importância para aperfeiçoamento na técnica cirúrgica e padronização das condutas frente a cada complicação, diminuindo assim a morbidade e a chance de falência cirúrgica a longo prazo. 


\begin{abstract}
Objective: To evaluate the joined precocious complications in surgeries of mitomycin trabeculectomy, carried through in residence service-school, aiming at better perfectioning in the technique and postoperative accompaniment of the patients. Methods: Retrospective study by means of record analysis. The patients evaluated amount sex, age and race. Was included patients with primary of angle open glaucoma and submitted the mitomycin trabeculectomy. A total of 43 patients had been analyzed, 25 were male and 18 female; 19 were black, and the age ranged of 51 the 82 years (average 68 years). The patients were evaluated in first and seventh postoperative days, or if necessary before first week. The complications in had been registered it and were: bleb leaking, shallow anterior chamber, atalamy; hifema, choroidal detachment. Results: The age average was of 68 years, $58.1 \%$ of the patients (25 in the total) was male and $44.1 \%$ were black. The Bleb leaking was the most frequent complication, representing $25.56 \%$ of surgeries and $36.66 \%$ of all the complications (11 patients). Shallow anterior chamber was observed in $17.5 \%$ of surgeries and $22.5 \%$ of complications (seven patients). Choroidal detachment was present in $14.6 \%$ of patients and $19.3 \%$ of the complications in 1 a week ( seven patients). Atalamy was found in three patients, represented of $6.97 \%$ of patients and $9.67 \%$ of the complications. Hifema also was observed in three patients, being all found they in the Immediate Postoperative Conclusion: The frequency of complications found in this work this inside of the limits observed in literature. Quantify the percentage of postoperative complications during the first week is of fundamental importance for improvements in intra operative and standardization of the behaviors front to
\end{abstract}

each complication, standardization of behavior in response to each complication, thus reducing the morbidity and chance of surgical failure in the long term.

Keywords: Glaucoma, open-angle/surgery; Postoperative complications; Trabeculectomy/efeitos adversos; Mitomycin/therapeutic use

\section{RefERÊNCIAS}

1. Hoskins HD, Kass M. Surgery to relieve outflow block: external filtering procedures, In: Hoskins HD, Kass M. BeckerShaffer's diagnosis and therapy of the glaucomas. St. Louis: Mosby; 1989. p.552-71.

2. Heuer DK, Parrish RK 2nd, Gressel MG, Hodapp E, Palmberg $\underline{\mathrm{PF}}, \underline{\text { Anderson DR. }}$ 5-fluorouracil and glaucoma filtering surgery. II. A pilot study. Ophthalmology. 1984; 91(4):384-94.

3. Chen CW. Enhanced intraocular pressure controlling effectiveness of trabeculectomy by local application of Mitomycin-C. Trans Asia-Pacific Acad Ophthalmol. 1983; 9:172-7.

4. Chen CW, Huang HT, Bair JS, Lee CC. Trabeculectomy with simultaneous topical application of mitomycin-C in refractory glaucoma. J Ocul Pharmacol. 1990; 6(3):175-82.

5. Skuta GL, Beeson CC, Higginbotham EJ, Lichter PR, Musch DC, Bergstrom TJ, et al. Intraoperative mitomycin versus postoperative 5-fluorouracil in high-risk glaucoma filtering surgery. Ophthalmology. 1992; 99(3):438-44.

6. Susana Júnior R. Cirurgia do glaucoma. São Paulo: Roca; 2001.

7. Susana Júnior R, Weinreb RN. Glaucoma: answers in glaucoma. Rio de Janeiro: Cultura Médica; 2005.

8. Lewis RA, Phelps CD. Trabeculectomy v thermosclerostomy. A five-year follow-up. Arch Ophthalmol. 1984; 102(4):533-6.

\author{
Endereço para correspondência \\ Dr. Gustavvo Carneiro Vilasboas Gutemberg \\ Rua Cananéia, nº 168 - Apto. 12 - Val Paraíso \\ Santo André (SP), Brasil
}

Tel: (11) 7736-5227 